\title{
Quick Response (QR) Code based Attendance Marking System
}

\author{
Dhruvil Shah \\ Department of Computers, \\ KJ Somaiya College of Eng. \\ Mumbai - 77, India
}

\begin{abstract}
Every classroom/lab/workshop has an attendance tracking system in them. Currently the ways in which one can take such attendance/presence marking is via manual calling of IDs (roll numbers in case of universities), fingerprint scanners or face detection mechanisms. But all these either require an infrastructure (scanners) or are too tedious. This paper puts forward a new technique of marking the attendance of students. It uses QR code mechanism, which is very common in payments and shipment tracking today, to mark attendance or presence.
\end{abstract}

\section{General Terms}

Faculty: The entity willing to register the attendance of students.

Student: The entity to be marked attended/present at some place typically classroom, lab, workshops, and community sessions

\section{Keywords}

Attendance decentralized QR-code on-demand-server

\section{INTRODUCTION}

Attendance marking and management is a very important and tedious task for universities. In most of the classrooms it is taken by manually calling out names or roll numbers which is prone to errors as there could be proxies, i.e., fake answering of this call by someone else. This is a security risk as there is a chance of masquerading attack on system. So instead one can utilize technology to take advantage of some unique identifiers within humans like fingerprints to mark attendance or presence. As mentioned in [1], largely there are two types of attendance marking frameworks:

\section{a. Manual Attendance System (MAS) \\ b. Automatic Attendance System (AAS)}

MAS is the traditional pen paper and calling based method. As mentioned it is prone to proxy errors. Also it is time consuming and does not scale well when the head count is large as is the case of universities with typical size of classrooms being 70 to 100 students.

So to reduce this workload one can use AAS. As the name suggests these are automatic systems and hence easy to manage. Typically these include a proprietary system with scanner which scans fingerprints, iris or other biometrics and a centralized server which manages these systems. These servers are a concern as they are a single point of failure. Although with the rise of cloud and micro service based architecture it is not that common for a server to fail. But it is still infrastructure that needs investment. Also major universities have a private network built and requires gateways to communicate with theses proprietary server.

Major research is going on in improving the scanner technology to make them as simple and powerful as possible but this paper dives into replacing the centralized aspect and infrastructure required to setup such systems with some more common, day-to-day technologies like cameras within smartphones.

\section{LITERATURE REVIEW}

There are various common attendance marking systems which are discussed here.

\subsection{Biometric based}

From [8], [9] and [10], it is clear that in these system the scanners read the basic elemental biometric data their systems rely on for e.g., segments from fingerprints, iris scans or others. These systems use the fact that humans have a built in uniqueness in their fingerprints and iris and take advantage of that to ensure that only the correct student is marked attended. In system security terms it is a mechanism which ensures entity authentication on the basis of something possessed by the user. These systems have a single device with embedded scanners in them and are generally portable. These devices capture the biometric and send the data to servers for further maintenance and storage. The issue is the cost which is on an average Rs. 25/person/month. For an average classroom of 100 students this cost goes to Rs. 2500/classroom/month and there could be $10 \mathrm{~s}$ to $50 \mathrm{~s}$ to $100 \mathrm{~s}$ of such classrooms in a decent university making the total cost around Rs. 25k to almost Rs. $250 \mathrm{~K}$ which is very expensive for just attendance sake. Also it can cause distraction during the lecture as students get distracted by these systems.

\subsection{RFID Based}

As mentioned in [7], the RFID based systems require that every user has an RFID tag embedded within their ID cards and students have to swipe them near the register for marking the attendance. These RFID based ID cards are issued by the universities and hence the university again bears the cost of infrastructure. Issue arises as this method is again prone to proxies as a student only needs another student's ID card to fool the system which becomes much easier than MASs

2.3.Machine Learning, aka, face recognition There is a huge surge in machine learning technology which gave rise to face recognition technology where companies are using face as a primary medium to authenticate an entity. From [2] and [6] it is clear that these devices analyze camera feed in frames and try to recognize the face and then match it with a user. There is a tremendous rise in using this type of technology for attendance marking system but the only issue is that the feed of classroom can have faces of let's say $40 \%$ from one feed of camera making it 40 students on an average. 
So the task is too expensive as now the face recognition algorithm has to first recognize 40 of these faces in contradiction to 1 and map these 40 faces to a student in database. These steps of recognition and mapping although sound simple are very difficult as accuracy of recognizing faces in such heavy feed can be affected drastically on the basis of models and sometimes it might even miss out students if it cannot identify their faces or map them accurately which implies that scaling is a big issue for such systems and similarly proxy also becomes easier as now one just has to show these cameras a photo of the student who is to be proxy.

\section{PROPOSED SYSTEM}

It is common for students and faculties to have mobile devices and these mobile devices are powerful. The system uses this aspect as an advantage and offer an application that runs on the devices of students and faculties for Attendance system. The system proposed uses the more common technology QR code which has seen rise since the rise of mobile based payment wallets, and is many times used for tracking shipments as well.

The system also relies on the fact that devices (at least in vicinity) have a next to 0 probability of having the same Media Access Control (MAC) Address.

In this system there are 2 main entities: faculty and a student.

Faculty starts a classroom that students can mark themselves as attended to. This is done using QR codes and on-demand HTTP servers on faculty's device. The student can use their mobile devices to scan the QR code and mark them present for that lecture

\subsection{Architecture}

The proposed solution is an application with 2 modes: viz faculty and student and runs on Wi-Fi network primarily. Fig 1 shows an overview of the proposed system.

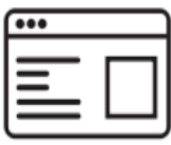

Application

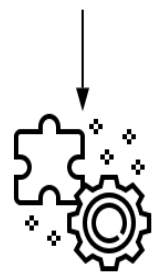

\section{Auto Detect IP, BSSID, SSID, MAC}

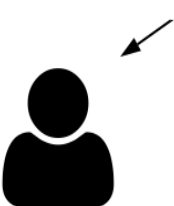

Faculty

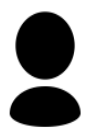

Student
Fig 1: System Architecture

\subsection{Auto-detection of IP, BSSID, SSID, MAC}

This component is responsible for fetching the IP(v4) address of the device it is running on, BSSID of the Wi-Fi Access Point (AP), SSID the access point's name and the device's MAC (media access control) Address for unicast mode.

\subsection{Faculty}

In faculty mode the first step, as shown in Fig 2, is to collect details about faculty and the lecture being conducted. This information is used ahead in the attendance sheet that will be saved on faculty's device. The faculty mode further has two complicated components in itself viz: QR code and OnDemand On-Device Server. The students who are marked attended will be stored in an Array (hereafter named studentsArray) and when the server is closed by faculty this studentsArray will be used to create the attendance sheet. The server also maintains a map which saves the MAC addresses of the students who are marked attended successfully for proxy detection.
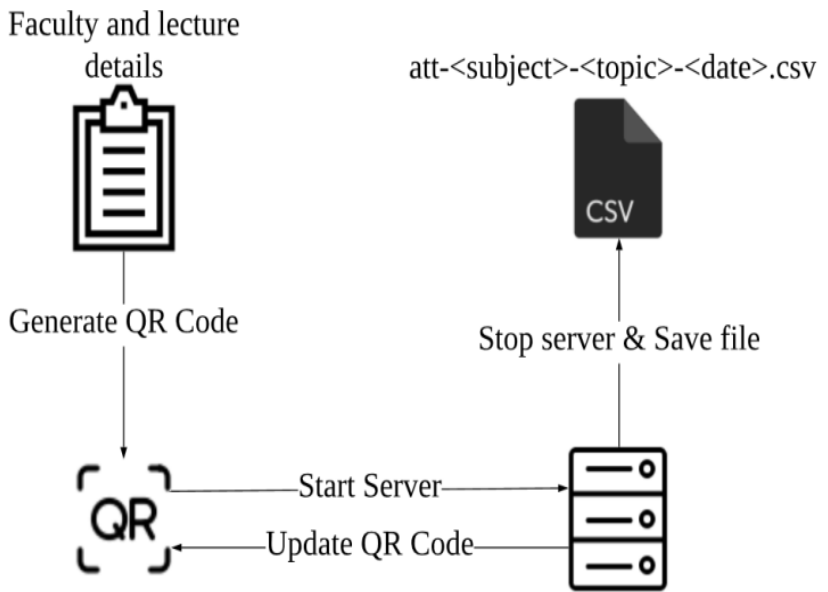

On-Demand on-device Server

Fig 2: Faculty Mode

\subsubsection{QR Code}

The algorithm used to generate $\mathrm{QR}$ code could be standard available with packages or libraries but the data we use to hide in it is important and a new encoding scheme is used for it (any encoding scheme not necessarily this can be used and corresponding decoding has to use on the student side of which some are mentioned in [4]) which is as follows

The length of the string who's QR code is to be generated has to be 128 characters long

- The string (hereafter called text) will be divided into several parts. Viz first salt, index (current length of studentArray), encoded IP, and encoded BSSID, SSID and second salt.

- All of these parts are then concatenated using "\#” and hence a constraint is that salts cannot contain "\#”.

- The encoding of IP and BSSID is done on the basis that they both contain a special character '.' and "."” respectively. We replace these with alphabets in orderly fashion, i.e., first '.' by ' $A$ ', second by 'B', third by ' $\mathrm{C}$ ' and so on. 
- So for e.g. consider

studentsArray: [],

IP: 192.168.0.101,

BSSID: 02:00:00:00:00:00,

SSID: "Test network". Then the text comes out to be:-

?\$[\$:+i0|+:^d*|[dih:\#0\#192A168B0C101D\#02H00I00J0 0K00L00M\#Test network\#\& w7384*]\{[\%/f\{:xz85rd^a

Note: characters E \& F are left out deliberately as BSSID uses A-F and hence it can lead to ambiguity to use them for encoding purposes

\subsection{2 on-Demand on-Device Server:}

This component is the heart of the application and the main part responsible for decentralization. This component uses the faculty device as a resource and runs a small "http" server on it while the faculty is willing to take attendance. The server is not meant for high performance as according to the architecture the faculty device (as explained ahead) should only receive one request for marking attendance at a time and today's modern devices are capable enough to do so. The component goes as shown in Fig.3.

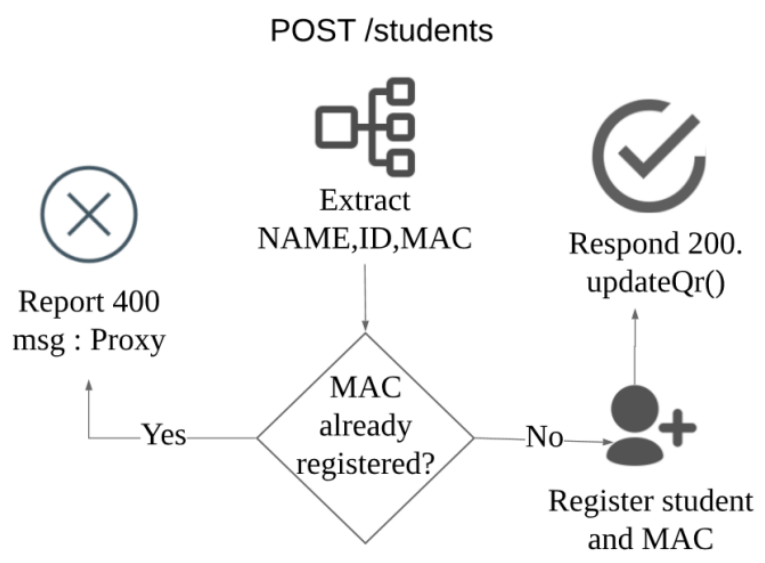

Fig 3: On-Demand On-device server overview

Each request on server has to have \{NAME,ID,MAC \} the following attributes in the body. The MAC address is checked for existence in MAC map, and if found it indicates that the server has already received an entry from the following MAC address and this could be an attempt to mark proxy for some student and hence report it. If the MAC does not exist in the MAC map then it is registered in the map, the student's details like NAME, ID (roll number) are combined as an object and appended to the studentsArray. After this as the index is new the QR code is to be updated using the new index. The index encoded in the QR code should always indicate the current length of the array. This helps in another form of proxy detection as students could just capture the QR code image and share among themselves but since the index is updated every time a student marks himself present the recorded QR code is no longer valid and hence cannot help mark proxy. But this also imposes a constraint that only one student should try to mark himself attended at a time otherwise a valid attendance might be regarded as proxy by the system.

After the work is done faculty can request to close the attendance and the application will save an attendance sheet into the faculty's device which can be used further for management. The name of the file saved is in the format: att-<subject $>-<$ topic $>-<$ date $>$.csv

where subject is the subject being taken, topic of the lecture and date on which the lecture was taken.

\subsection{Student}

The student mode is rather much simpler and just has two phases (refer Fig 4).

\subsubsection{Scanning}

Scan the QR code, read the text, split at "\#” and extract index, encoded IP, encoded BSSID and SSID. The BSSID and SSID are used to ensure that the student and faculty are on the same network and if not the student has to ensure the same. The IP address will be decoded and then it will be used to make a POST request as this is the IP address of the server (faculty device) on which the attendance service will be running

\subsubsection{Request}

In this phase the actual request is made to the decodedIP address with the body containing (NAME, ID (roll number), MAC \}. The response from the server is then displayed to the student which could be getting marked as attended or proxy being interpreted.

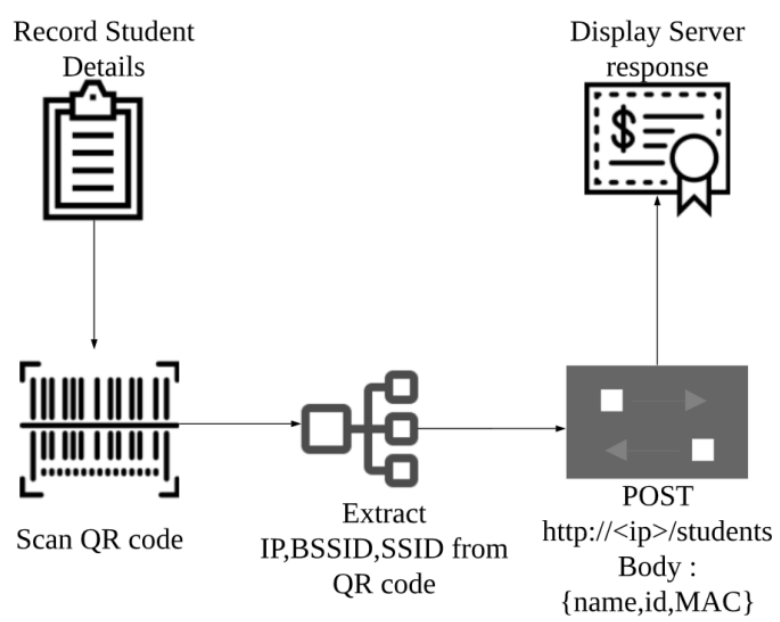

Fig 4: Student mode

\section{IMPLEMENTATION AND CONSTRAINTS}

Hardware Requirements:

- 2 GB RAM (minimum), 4 GB or more (recommended) (Although application does not require all that memory it safe for the device to have some extra memory for OS and background tasks).

- Storage: $100 \mathrm{MB}$ for application and $50 \mathrm{MB}$ for attendance sheets. Total $150 \mathrm{MB}$.

Software Requirements:

- iOS: 10 and above

- Android: 6(Marshmallow) and above

The application in [11] is developed using react-native framework so that it can be used across different platforms. The framework has several packages and libraries that help with QR code generation, scanning and deploying on-demand on-device server as well.

The system can easily be used by any faculty is that the above system can be used to track attendance of classroom, labs, 
sessions and such presence marking cases. Table 1 below shows a comparison of the proposed system against the

existing systems.

Table 1: Comparison with existing systems

\begin{tabular}{|c|c|c|c|c|c|c|}
\hline & \multicolumn{2}{|c|}{ Cost (to administration) } & Accuracy & Scaling & Time of operation & $\begin{array}{c}\text { Security(against } \\
\text { proxy) }\end{array}$ \\
\cline { 2 - 6 } & Devices & Operational & & & & \\
\hline Biometric & High & Low & Very high & Normal & Very high & Very strong \\
\hline RFID & Very high & Moderate & Very high & Easy & High & Poor \\
& & & & & & Poor \\
\hline Face recognition & Moderate & High & Moderate to low & Difficult & Low & Very Strong \\
\hline Proposed System & Low & High & Very High & Easy & Very high & \\
& & & & & & \\
\hline
\end{tabular}

But the system has the following constraints:

- Same Network: Both the faculty and students have to be on the same network for this application to work without any internet (outside the private network resources).

- Serial Attendance: Only one student at a time can mark himself present because of the security of the system to prevent proxies.

- Acquiring MAC Address: An issue that arises in implementing the above system is that some vendors block any application from acquiring information about device's MAC address but this is resolved by using the device ID assigned by the vendor to that device and is expected to be unique

\section{CONCLUSION AND FUTURE WORK}

\subsection{Conclusion}

The above system decreases the cost to the university for tracking attendance as the system uses devices that the faculties and students already own and convert them into resources for attendance system and with rise in smartphone market it can ensured that this system is feasible with almost 3 billion smartphones in the world and the count increasing. It is also a simple system where in a faculty has to just place a device in the room and every student entering it can scan it mark themselves present without causing much distraction.

\subsection{Future Work}

\subsubsection{Integration to university systems}

Many universities have some system for the management of its tasks like result display, lab experiments and attendance as well. Currently this system does not have any way to integrate it directly to the university system making the management part more easy and automated

\subsubsection{Security of the servers}

Currently there is no method for ensuring the security of the requests that are made and anyone can make requests to the server with fake credentials and break its integrity. This security feature can be implemented by using encryption and hiding the keys inside the QR code which although available for all can only be understood by the application by means of encoding.

\section{REFERENCES}

[1] Nandhini R, Duraimurugan N and S.P.Chokkalingam, "Face Recognition Based Attendance System", International Journal of Engineering and Advanced Technology (IJEAT), Volume-8, Issue-3S, February 2019

[2] S. Sawhney, K. Kacker, S. Jain, S. N. Singh and R. Garg, "Real-Time Smart Attendance System using Face Recognition Techniques", 2019 9th International Conference on Cloud Computing, Data Science \& Engineering (Confluence), Noida, India, 2019, pp. 522525 .

[3] Mahansaria D. et al. (2010) An Automated and FullProof Attendance Marking Scheme (TATTEND) Using Three Integrated Technologies. In: Meghanathan N., Boumerdassi S., Chaki N., Nagamalai D. (eds) Recent Trends in Networks and Communications. WeST 2010, VLSI 2010, NeCoM 2010, ASUC 2010, WiMoN 2010. Communications in Computer and Information Science, vol 90. Springer, Berlin, Heidelberg

[4] Atul Hole, Mangesh Jadhav, Shivkant Kad, Swanand Shinde and Prof. Pramod Patil, "Encryption and Decryption of Data Using QR Authentication System", International Journal of Computer Science and Mobile Computing, Vol.3 Issue.4, April- 2014, pg. 488-496

[5] Chauhan Hardika, Dr. Nehal Chitaliya. "Smart Attendance Management and Analysis with Signature Verification." International Journal of Advance 
Research, Ideas and Innovations in Technology 3.3 (2017). www.IJARIIT.com.

[6] Abhilasha Varshney, Sakshi Singh, Suneet Srivastava, Suyash Chaudhary, Tanuja. "Automated Attendance System Using Face Recognition", International Research Journal of Engineering and Technology, Volume 06 Issue 05, May 2019, pg 7118 - 7123.

[7] Arulogun O. T., Olatunbosun, A., Fakolujo O. A., and Olaniyi, O. M. "RFID-Based Students Attendance Management System", International Journal of Scientific \& Engineering Research Volume 4, Issue 2, February2013

[8] L.S. Ezema, C.K.A. Joe-Uzuegbu, J. N. Eneh and I. Amanze. "FINGERPRINT BASED ATTENDANCE MANAGEMENT SYSTEM", International Journal of
Scientific \& Engineering Research, Volume 6, Issue 7, July-2015

[9] Hitesh Walia, Neelu Jain. "Fingerprint Based Attendance Systems-A Review", International Research Journal of Engineering and Technology (IRJET) Vol 3 Issue 5, May 2016, pg 1166 - 1171

[10] Khatun, Amena \& Haque, A.K.M Fazlul \& Ahmed, Sabbir \& Rahman, Mohammad. (2015). Design and Implementation of Iris Recognition Based Attendance Management System. 10.1109/ICEEICT.2015.7307458.

[11] AttendiFi: https://github.com/NightWing1998/AttendiFi

[12] Cost guide for biometric scanners, https://blog.mantratec.com/biometric-time-attendancesystem-cost 